TECHNICAL NOTE

\author{
S.M. Mirsattari \\ L.M. Tapsell \\ J.R. Ives \\ D.H. Lee
}

\section{MR Imaging-Compatible Electroencephalography Electrode System for an Epilepsy Monitoring Unit}

SUMMARY: We studied the usefulness of an MR imaging-compatible electroencephalography (EEG) electrode system for continuous EEG recordings in our epilepsy monitoring unit (EMU) by comparing 100 consecutive patients with MR imaging-compatible and MR imaging-incompatible EEG recording electrodes who underwent MR imaging between 3:00 PM and 7:00 AM. The MR imaging-compatible system captured seizures in $21 / 50(42 \%)$ patients and clinically valuable new electrographic data in $13 / 50(26 \%)$ patients during the study interval, whereas possible seizures were lost to recording in $19 / 50$ (38\%) patients in the MR imaging-incompatible system. EEG recording was comparable by both systems, but the nurses could disconnect and reconnect the patients to their electrode cables only in the MR imaging-compatible system during the study interval while the EEG technologists were off duty. This study shows that the MR imaging-compatible system could be used routinely for long-term monitoring of the patients in EMUs.
A lthough electroencephalography (EEG) remains the primary tool in the investigation of patients with epilepsy, MR imaging studies are essential for the evaluation of patients with medically intractable epilepsy. ${ }^{1}$ For patient safety ${ }^{2}$ and to avoid distortion of the images, EEG electrodes are routinely removed before MR imaging of patients admitted to the epilepsy monitoring units (EMUs) and are re-applied afterward. However, several studies have demonstrated that safe and distortion-free MR images can be obtained with EEG disk electrodes left in place. ${ }^{3-6}$ In addition, it has been shown that the removal of the EEG electrodes with collodion remover/acetone and their re-application immediately afterward can result in scalp abrasions, pain, and rare infection with blood-borne pathogens such as human immunodeficiency virus or hepatitis. $^{7}$

The 8-bed EMU at London Health Sciences Centre (LHSC) in London, Ontario, is a referral center that admits patients from all geographic parts of Canada. The routine imaging sequences used for epilepsy work-up at LHSC include a 3D plane localizer; sagittal T1, axial spin-echo, and axial diffusionweighted echo-planar imaging; coronal short $\mathrm{T} 1$ inversion recovery, coronal fluid-attenuated inversion recovery, coronal $\mathrm{T} 2$, and inversion recovery prepped 3D spoiled gradient echo imaging; and a high-resolution axial T1-weighted anatomic study with 1.0 - to $1.5-\mathrm{mm}$ section thickness (approximately 180 sections) and 0 gap for subsequent CT-MR imaging superimposition to visualize the intracranial electrodes in cases that require invasive EEG monitoring. ${ }^{8}$ Frequently one of our routinely used MR imaging sequences is missing from the MR imaging studies performed in other centers so that most patients who are admitted to our EMU undergo MR imaging at our center. Moreover, depending on the findings of videoEEG telemetry in a given patient and clinical circumstances,

Received and accepted December 11, 2007

From the Departments of Clinical Neurological Sciences (S.M.M., J.R.I.), Medical Biophysics (S.M.M.), and Diagnostic Radiology and Nuclear Medicine (S.M.M., D.H.L.), The University of Western Ontario, London, Ontario, Canada; London Health Sciences Centre (S.M.M., J.R.I. D.H.L.), London, Ontario, Canada; and Epilepsy Clinic (L.M.T.), Mayo Clinic Hospital, Scottsdale, Ariz.

Please address correspondence to Seyed M. Mirsattari, MD, PhD, FRCP (C), B10-108, London Health Sciences Centre, 339 Windermere Rd, London, Ontario, N6A 5A5 Canada; e-mail: smirsat2@uwo.ca

DOI 10.3174/ajnr.A1143 additional MR imaging studies by using surface coil or dedicated MR imaging sequences such as gradient-echo or MR angiography may be required to rule out focal cortical dysplasia, hemosiderin deposition, or vascular abnormality as the cause of seizures. We also perform functional MR imaging (fMRI) and MR spectroscopy in most patients. Therefore, almost all the patients who are admitted to our EMU require at least $1 \mathrm{MR}$ imaging study during their hospital stay.

Unpredictable timing of the in-patient MR imaging at our center and potential scheduling conflicts between the MR imaging and EEG technologists used to result in significant time lost in EEG recording by the MR imaging-incompatible system. Moreover, disconnection, removal, and re-application of the EEG electrodes require an EEG technologist whose availability has to be determined in conjunction with the operating hours of the MR imaging scanner. Given that many of the MR imaging studies for EMU patients in our center are done during the off-time of EEG technologists, patients requiring continuous EEG (cEEG) recording may not be monitored for a considerable amount of time using the MR imaging-incompatible system. The purpose of this study was to evaluate the usefulness of the MR imaging-compatible EEG electrode system in a large (ie, 8-bed) EMU and compare it with the MR imaging-incompatible system.

\section{Technique}

The MR imaging-incompatible EEG electrodes at our center are made of tin that contains paramagnetic material (ie, nickel). Our MR imaging-compatible EEG electrodes are made of gold-plated copper and silver wires, whose assembly into the MR imaging-compatible EEG disk electrode system is described in detail in a previous publication. ${ }^{6}$ This setup permits artifact-free imaging of the patients' heads in a $1.5 \mathrm{~T}$ MR imaging scanner with the EEG electrodes attached. The electrode connectors used in this setup can be easily disconnected and reconnected by the nursing personnel. Figures 1 and 2 show examples of this setup. Therefore, with the MR imaging-compatible EEG electrode system, the long-term monitoring of EEG in the EMU was interrupted the least.

We compared the recording characteristics of 50 consecutive patients with the MR imaging-compatible EEG electrode system with the recording characteristics of 50 consecutive patients using the MR imaging-incompatible EEG system. The MR imaging-compatible group was among the first 100 patients who used this system between 


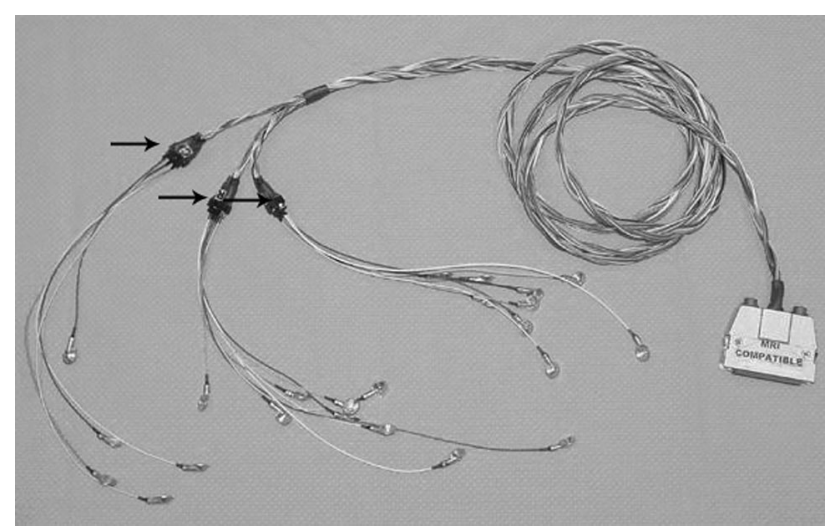

Fig 1. MR imaging-compatible EEG disk electrodes made of gold-plated copper and silver wires attached to an MR imaging-compatible interface EEG cable via 3 connectors (arrows). The cable was disconnected before the MR imaging.

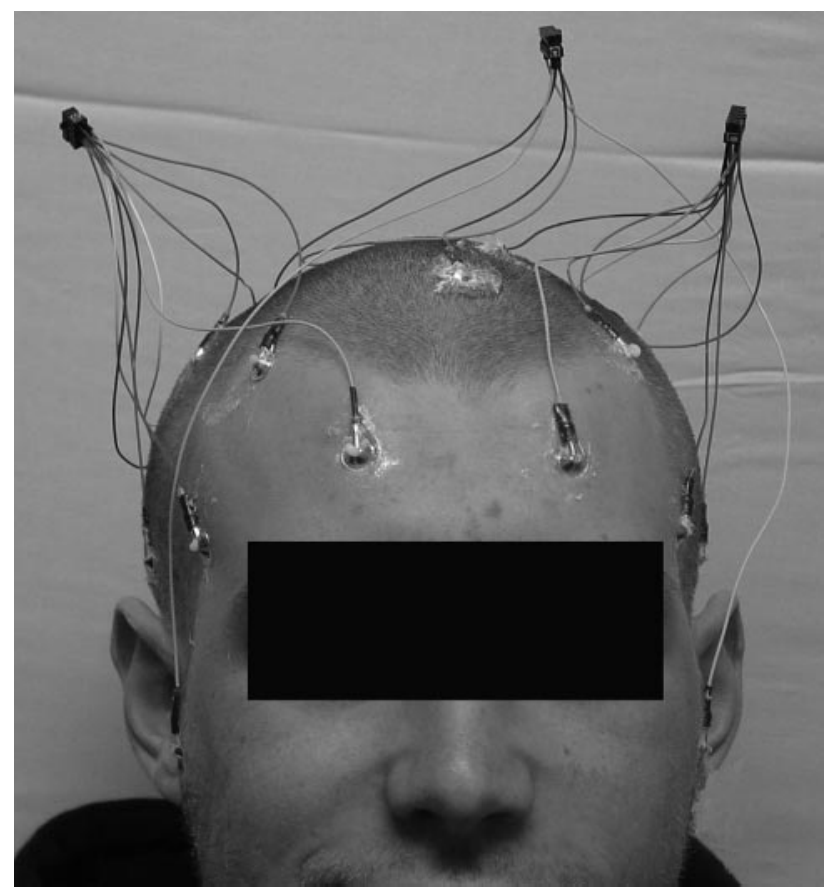

Fig 2. Attachment of the MR imaging-compatible EEG electrodes to the scalp in the extended international $10-20$ system.

March 2003 and February 2004. Only patients who underwent MR imaging between 3:00 PM and 7:00 AM (ie, they returned to the EMU after EEG technologists' regular working hours) were included in this study. Fifty patients in this group had undergone their MR imaging studies outside the study interval and were excluded from further analysis. The patients monitored with the MR imaging-incompatible EEG system were enrolled between January 2002 and December 2002. This interval preceded the use of the MR imaging-compatible EEG recording system at our center.

Written consent was obtained before MR imaging from the patients who used the MR imaging-compatible EEG recording electrodes. All MR imaging was performed in a 1.5T Signa Excite scanner (GE Healthcare, Milwaukee, Wis). We analyzed the number of seizures or nonepileptic events (NEEs) missed on recording in either group because of MR imaging; recorded seizures (clinical or electrographic) and NEEs; first recorded seizure; clinically relevant new electrographic data (ie, epileptic seizures or interictal epileptic spikes); and the only recorded seizure in the hospital during the study interval. The length of the hospital stay, the number of times that EEG technologists were called back to set up the EEG electrodes after regular work hours, major scalp abrasions caused by the EEG electrodes requiring a delay in recording, quality of visual EEG display as judged by the electroencephalographers, and the duration of EEG recording intervals were also analyzed.

A 2-tailed Student $t$ test was used to determine the impact of the MR imaging-compatible EEG system on the length of hospital stay. All the EEG recordings were done digitally by using 23 electrodes placed according to the extended international 10-20 system of EEG electrodes ${ }^{9}$ plus 2 mandibular notch electrodes (M1 and M2; XLTEK, Oakville, Ontario, Canada). The mean age of the study patients was $35 \pm 14$ years in the MR imaging-compatible group and $34 \pm 14$ years in the MR imaging-incompatible group. There were 27 women in the first group and 24 in the second group.

\section{Discussion}

The 2 study groups were similar with respect to the duration of the study and age and sex of the study patients. It took approximately 1 year to collect the data in each group. Twenty-one of $50(42 \%)$ patients with the MR imaging-compatible EEG electrodes had recorded seizures or NEEs during the study period, which included seizures in $17 / 50(34 \%)$ patients, NEEs in 4/50 (8\%) patients, and first recorded seizure in $9 / 17(53 \%)$ patients. In $13 / 50(26 \%)$ patients in this group, clinically valuable new data became available during the study interval. MR imaging did not result in missed seizures in this group. Nineteen of $50(38 \%)$ patients with the MR imaging-incompatible EEG electrodes had presumed seizures not recorded during the MR imaging timeframe, including first seizure in the EMU in $5 / 19(26 \%)$. One of $50(2 \%)$ patients in this group had his first and only seizure during his EMU stay while the EEG electrodes were off for MR imaging. The average length of hospital stay was 9 and 11 days for the patients with the MR imagingcompatible EEG recording electrodes and those with MR imaging-incompatible EEG electrodes, respectively $(P=.0003)$.

There were additional qualitative advantages of the MR imaging-compatible EEG recording electrodes over those that were MR imaging-incompatible. The EEG technologists did not have to return to the EMU after their regular work hours to set up the electrodes for the patients who had the MR imaging-compatible EEG recording electrodes. For monetary reasons, this was not demanded of the EEG technologists for the MR imaging-incompatible system either. None of the MR imaging-incompatible EEG electrodes were re-applied till the following day, resulting in missed data for a minimum of 10 hours in a given patient (maximum, 18 hours). Only a maximum of 1 hour of cEEG recording was lost to scanning in the MR imaging-compatible group. There were no major scalp abrasions in either group to interfere with cEEG recordings. The MR imaging-incompatible electrodes were temporarily removed and re-applied as per usual practice every 3 days, whereas the MR imaging-compatible EEG electrodes were reapplied every 4 days. The additional day of recording was permitted for the MR imaging-compatible EEG electrodes because none of the patients requested their earlier removal and there were no other indications for re-applying them earlier despite their daily inspections by the EEG technologists.

The MR imaging-compatible electrodes provided compa- 
rable EEG recordings as judged by the visual display of the traces on a computer screen with fewer artifacts because they were left on the scalp longer without periodic removal and re-application. None of these records were classified as "technically unsatisfactory" by the electroencephalographers caring for the patients. The MR imaging-compatible design was less cumbersome for the patients because they were assembled in a harness fashion and were not individual wires. Leaving the MR imaging-compatible EEG electrodes on the patient's head reduced the patient's fear of missed seizures because the EEG electrodes stayed on the head without the risk of missing data during the MR imaging timeframe. It eliminated MR imaging/ EEG scheduling conflicts. The EMU nursing staff was fully capable of disconnecting/reconnecting the electrodes from the patient's electrode cables for MR imaging. The harness design was color-coded to ensure quick, accurate, and easy disconnection and reconnection.

The MR imaging-compatible EEG recording electrode system was efficacious when compared with the MR imagingincompatible EEG system in all aspects of the study parameters. It led to capturing more seizures, NEEs, or new data in the EMU. It eliminated the need for re-applying the electrodes while making all the patients in the EMU available for an MR imaging study without significantly interrupting their EEG recordings. A shorter hospital stay in this group was another potentially useful feature of this system, but other factors such as the type of epilepsy might have accounted for it. These patients were also available for additional studies such as fMRI and MR spectroscopy. Therefore, this study supports the findings of an earlier study of a 0.5T MR imaging scanner ${ }^{3}$ and our previous study of a $1.5 \mathrm{~T}$ MR scanner ${ }^{6}$ : Modified EEG recording electrodes can safely be left on the scalp during MR imaging studies.

This study demonstrates the long-term use of a new clinical electrode system meant to better use resources in a busy EMU. To date, more than 1000 patients at our center have undergone MR imaging with these MR imaging-compatible EEG disk electrodes in place. There have been no safety concerns or any susceptibility/artifact problems requiring a repeat of the MR imaging study. All patients who have been admitted to the EMU at LHSC since March 2003 have used these MR imaging-compatible EEG disk electrodes. Therefore, the MR imaging-compatible EEG recording electrode system is useful for cEEG monitoring in busy EMUs that use 1.5T MR scanners. The reversible and nonreversible changes that have been associated with seizures ${ }^{10-12}$ make this EEG recording system a valuable tool in any EMU to better understand the potential consequences of seizures. We have evaluated the safety of this EEG electrode system in a Unity Inova 4T whole-body MR imaging scanner (Varian, Palo Alto, Calif) with a Sonata gradient coil (Siemens, Erlangen, Germany). ${ }^{13}$ Finally, all the components of our MR imaging-compatible EEG electrode system are commercially available and can easily be assembled. $^{6}$

\section{Conclusion}

Because MR imaging is increasingly used in the EMUs, we propose that MR imaging-compatible EEG electrodes should be used routinely in patients who require cEEG monitoring. EMUs that are equipped with dedicated MR imaging scanners or have unlimited access to them or those that serve as regional facilities may not benefit greatly from our setup because their patients can be scanned before or after the completion of video-EEG telemetry. Despite being safe in a CT scanner, these MR imaging-compatible EEG electrodes cause artifacts and distortion of the CT images. Our results may, therefore, not be applicable to all EMUs, but they are applicable to those EMUs that rely on 1.5T MR imaging for the examination of patients with medically intractable epilepsy.

\section{References}

1. Duncan J. The current status of neuroimaging for epilepsy: editorial review. Curr Opin Neurol 2003;16:163-64

2. Roth BJ, Pascual-Leone A, Cohen LG, et al. The heating of metal electrodes during rapid-rate magnetic stimulation: a possible safety hazard. Electroencephalogr Clin Neurophysiol 1992;85:116-23

3. Lufkin R, Jordan S, Lylyck P, et al. MR imaging with topographic EEG electrodes in place. AJNR Am J Neuroradiol 1988;9:953-54

4. Ives JR, Warach S, Schmitt F, et al. Monitoring the patient's EEG during echo planar MRI. Electroencephalogr Clin Neurophysiol 1993;87:417-20

5. Lemieux L, Allen PJ, Krakow K, et al. Methodological issues in EEG-correlated functional MRI experiments. Int J Bioelectromagnetism 1999;1:87-95

6. Mirsattari SM, Lee DH, Jones D, et al. MRI compatible EEG electrode system for routine use in the epilepsy monitoring unit and intensive care unit. Clin Neurophysiol 2004;115:2175-80

7. Ferree TC, Luu P, Russell GS, et al. Scalp electrode impedance, infection risk, and EEG data quality. Clin Neurophysiol 2001;112:536-44

8. Steven D, Parrent A, Wiebe S, et al. Automated fusion technique for fusion of MRI and CT for visualization of subdural electrodes. Can J Neurol Sci 2005; 32( suppl 1):S75

9. Jasper HH. The ten-twenty electrode system of the International Federation. Electroencephalogr Clin Neurophysiol 1958;10:371-75

10. Mirsattari SM, Lee DH, Jones MW, et al. Transient lesion in the splenium of the corpus callosum in an epileptic patient. Neurology 2003;60:1838-41

11. Bauer G, Gotwald T, Dobesberger J, et al. Transient and permanent magnetic resonance imaging abnormalities after complex partial status epilepticus. Epilepsy Behav 2006;8:666-71

12. Korngut L, Young GB, Lee DH, et al. Irreversible brain injury following status epilepticus. Epilepsy Behav 2007;11:235-40

13. Stevens TK, Mirsattari SM, Ives JR, et al. Evaluation of MR compatibility for EEG electrodes. Epilepsia 2004;45(suppl 7):S304 